Proyecciones

Vol. 24, No 3, pp. 239-255, December 2005.

Universidad Católica del Norte

Antofagasta - Chile

\title{
THE NATURAL VECTOR BUNDLE OF THE SET OF MULTIVARIATE DENSITY FUNCTIONS
}

\author{
E. MARCHI \\ and \\ P. M. MORILLAS \\ UNSL - CONICET, ARGENTINA \\ Received: July 2005. Accepted : November 2005
}

\begin{abstract}
We find a description of the set of multivariate density functions with given marginals and introduce an associated vector bundle. The interest for the probability theory is restricted to the nonnegative elements in the sets of the derived vector bundle. The fiber is the space of all correlation measures among a multivariate density function and its unidimensional marginals.
\end{abstract}

Keywords : Multivariate density function, marginals, vector bundle

Subjclass 2000 : Primary 60E05

*Research partially supported by CONICET, Argentina. 


\section{Introduction}

An important branch in probability and statitics is the study of distributions with given marginals. This study was initialized by Wassily Hoeffding (1940, 1941) and Maurice Fréchet (1951). Since then, its theoretical and applied development have been remarkable (for an account of the first steps in this development see Dall'Aglio (1991)). When the marginals are unidimensional, the most important tool in this study are copulas. Copulas were introduced by Abe Sklar (1959) in response to a query of Fréchet. Sklar proved that if $H$ is the joint distribution function of $n$ random variables $X_{1}, \ldots, X_{n}$, and $F_{1}, \ldots, F_{n}$ are the distribution functions of $X_{1}, \ldots, X_{n}$, respectively, then there exists an $n$-copula $C$ such that $H\left(x_{1}, \ldots, x_{n}\right)=C\left(F_{1}\left(x_{1}\right), \ldots, F_{n}\left(x_{n}\right)\right)$. The $n$-copula is uniquely determined on $\operatorname{range}\left(F_{1}\right) \times \ldots \times \operatorname{range}\left(F_{n}\right)$, so that $C$ can be thought of as a description of the way in which a joint distribution function is related to its one-dimensional marginals. Excellent references about copulas are Joe (1997) and Nelsen (1999).

Independently of the above mentioned development and using a geometrical approach, Ezio Marchi $(1969,1972)$ studied the set of discrete multivariate density functions with given unidimensional marginals which it motivates a great variety of new concepts of cooperative equilibrium in game theory (see Marchi $(1976,1986 \mathrm{a}, 1986 \mathrm{~b}, 1988,1992,1996)$ and Marchi, García Jurado and Prada (1991)). He first studied the bivariate case concerning to some problems about bimatrix zero sum games. Then, he studied the multivariate case introducing a natural vector bundle.

In particular, Marchi (1972) showed by considering finite sets $X_{i}, i=$ $1, \ldots, n$, that the Cartesian product $\widetilde{X}_{N}$ of the sets of density functions $\widetilde{X}_{i}$ defined on $X_{i}, i=1, \ldots, n$, is naturally embedded in the set $\widetilde{X_{N}}$ of the density functions defined on the Cartesian product $X_{N}$ of the sets $X_{i}$. Moreover, he derived $\widetilde{X_{N}}$ as a vector bundle having as the underlined manifold the image of the sets $\widetilde{X}_{i}$ under the natural embedding. The interest for the probability theory is restricted to the nonnegative elements in the sets of the derived vector bundle. Specifically, the base of the vector bundle contains the set $\widetilde{X}_{N}$ and the space contains the set of tensor products of elements in $\widetilde{X}_{i}, i=1, \ldots, n$. If $g_{i} \in \widetilde{X}_{i}, i=1, \ldots, n$, then the set of functions in $\widetilde{X_{N}}$ with unidimensional marginals $g_{i}$ consists of the nonnegative elements of the fiber in the vector bundle at the tensor product $\otimes_{i=1}^{n} g_{i}$. Moreover, any element of this set can be written as the sum of $\otimes_{i=1}^{n} g_{i}$ and an element of the type fiber of the vector bundle. So the fiber plays an 
important role, namely that of being the space of all correlation measures among a multivariate density function and its unidimensional marginals.

In this paper we extend the results obtained by Marchi (1972) considering $\sigma$-finite measure spaces $\left(X_{i}, \mu_{i}\right), i=1, \ldots, n$. It is worth noting that in the papers just mentioned, Marchi, in order to prove the results, considered extreme points and introduced basis which are not available in the general setting of arbitrary measure spaces. Here we use decompositions for the different involved spaces simplifying considerably the proofs of the different results.

The rest of the paper is organized as follows. In section 2 we obtain a decomposition of $L^{1}\left(X_{N}, \mu_{N}\right)$ where $\left(X_{N}, \mu_{N}\right)$ denotes the product measure space corresponding to the measure spaces $\left(X_{i}, \mu_{i}\right), i=1, \ldots, n$. We express $L^{1}\left(X_{N}, \mu_{N}\right)$ as a topological sum of tensor products of some subspaces of $L^{1}\left(X_{i}, \mu_{i}\right), i=1, \ldots, n$. In section 3 we obtain a description of the set of density functions in $L^{1}\left(X_{N}, \mu_{N}\right)$ with given unidimensional marginals and, in section 4 , we introduce the vector bundle. Finally, some concluding remarks are given in section 5 .

\section{A decomposition of $L^{1}\left(X_{N}, \mu_{N}\right)$.}

If $A$ is a finite set, then $|A|$ denotes the number of elements of $A$. We denote with $\mathbf{N}$ and

$\mathbf{R}$ the set of natural numbers and the set of real numbers, respectively. If $n \in \mathbf{N}$, then $N=\{1, \ldots, n\}$. To avoid ambiguities we assume that $n \geq 2$. If $K=\left\{k_{1}, \ldots, k_{m}\right\} \subseteq N$ with $k_{1}<\ldots<k_{m}$, then $\left(x_{K}\right)=\left(x_{k_{1}}, \ldots, x_{k_{m}}\right)$. If $S_{1}, \ldots, S_{n}$ are sets, then $S_{K}=S_{k_{1}} \times \ldots \times S_{k_{m}}$.

If $S$ is a subset of a vectorial space, then $\langle S\rangle$ is the linear space generated by $S$. If $E$ is a subset of a normed vectorial space $X$, then $c l E$ is the closure of $E$ in $X$. If $X_{i}, i \in N$, are normed vectorial spaces with norms $\|\cdot\|_{i}$, then $X_{N}$ is equipped with the product topology defined by any of the equivalent norms $\left\|\left(x_{N}\right)\right\|_{N}=\max \left(\left\|x_{i}\right\|_{i}: i \in N\right)$, or $\left\|\left(x_{N}\right)\right\|_{N}=\sum_{i=1}^{n}\left\|x_{i}\right\|_{i}$ for all $\left(x_{N}\right) \in X_{N}$. The symbol $\oplus$ denotes the topological sum.

In this paper we use some notions from measure and integral theory. For Fubini's theorem to be valid, all the measure spaces are considered to be $\sigma$-finite. If $f \in L^{1}(X, \mu)$, then $\|f\|_{(X, \mu)}$ is the norm of $f$. We consider the subspace of $L^{1}(X, \mu), L_{0}^{1}(X, \mu)=\left\{f \in L^{1}(X, \mu): \int_{X} f d \mu=0\right\}$. If $\left(X_{i}, \mu_{i}\right)$, $i \in N$, are $\sigma$-finite measure spaces and $\left(X_{N}, \mu_{N}\right)$ is the corresponding product measure space, we set $\int_{X_{K}} f d \mu_{K}=f$ for $f \in L^{1}\left(X_{N}, \mu_{N}\right)$ and $K=\emptyset$. 
Theorem 2.1. 2.1. Let $g \in L^{1}(X, \mu)$ be such that $\int_{X} g d \mu=1$. Then $L^{1}(X, \mu)=L_{0}^{1}(X, \mu) \oplus\langle g\rangle$.

Proof. Let $\sigma$ be the continuous function defined by

$$
\begin{gathered}
\sigma: L_{0}^{1}(X, \mu) \times\langle g\rangle \rightarrow L^{1}(X, \mu) \\
(z, c g) \rightarrow z+c g . .
\end{gathered}
$$

The function $\sigma$ is one to one and onto. In effect, if $f \in L^{1}(X, \mu)$, we can write $f=z+c g$ with $c=\int_{X} f d \mu$ and $z=f-c g \in L_{0}^{1}(X, \mu)$. This shows that $\sigma$ is onto. Suppose now that

$$
z_{1}+c_{1} g=z_{2}+c_{2} g
$$

with $z_{1}, z_{2} \in L_{0}^{1}(X, \mu), c_{1}, c_{2} \in \mathbf{R}$. Integrating the previous equality on $X$, and taking into account that $\int_{X} z_{1} d \mu=\int_{X} z_{2} d \mu=0$ and $\int_{X} g d \mu=1$, we obtain $c_{1}=c_{2}$, and then $z_{1}=z_{2}$. Thus $\sigma$ is one to one.

By the open map theorem, since $L_{0}^{1}(X, \mu)$ and $\langle g\rangle$ are closed subspaces of $L^{1}(X, \mu)$, then $\sigma$ is a topological isomorphism, or which is the same, the subspaces $L_{0}^{1}(X, \mu)$ and $\langle g\rangle$ decompose $L^{1}(X, \mu)$ in a topological sum.

Given $n$ real functions $f_{i}: X_{i} \rightarrow \mathbf{R}, i \in N$, where each $X_{i}$ is an arbitrary set, the tensor product of the functions $f_{i}$ is the function $\otimes_{i=1}^{n} f_{i}: X_{N} \rightarrow \mathbf{R}$ given by $\otimes_{i=1}^{n} f_{i}\left(x_{1}, \ldots, x_{n}\right)=f_{1}\left(x_{1}\right) \ldots f_{n}\left(x_{n}\right)$. Given $n$ closed subspaces $E_{i} \subseteq L^{1}\left(X_{i}, \mu_{i}\right), i \in N$, the tensor product of the spaces $E_{i}$ is $\otimes_{i=1}^{n} E_{i}=$ $c l\left\langle\left\{\otimes_{i=1}^{n} f_{i}: f_{i} \in E_{i}, i \in N\right\}\right\rangle$. For $i \in N$, let $E_{i}, F_{i} \subseteq L^{1}\left(X_{i}, \mu_{i}\right)$ be closed subspaces, $f_{i} \in E_{i}, g_{i} \in F_{i}$ and $K=\left\{k_{1}, \ldots, k_{m}\right\} \subseteq N$ with $k_{1}<\ldots<k_{m}$, then we set

$$
\begin{aligned}
\otimes_{k \in K} f_{k} \otimes_{k^{\prime} \in N \backslash K} g_{k^{\prime}} & =g_{1} \otimes \ldots \otimes g_{k_{1}-1} \otimes f_{k_{1}} \otimes g_{k_{1}+1} \otimes \ldots \otimes g_{k_{m}-1} \otimes \\
& \otimes f_{k_{m}} \otimes g_{k_{m}+1} \otimes \ldots \otimes g_{n},
\end{aligned}
$$

and

$$
\begin{aligned}
\otimes_{k \in K} E_{k} \otimes_{k^{\prime} \in N \backslash K} F_{k^{\prime}} & =F_{1} \otimes \ldots \otimes F_{k_{1}-1} \otimes E_{k_{1}} \otimes F_{k_{1}+1} \otimes \ldots \otimes F_{k_{m}-1} \otimes \\
& \otimes E_{k_{m}} \otimes F_{k_{m}+1} \otimes \ldots \otimes F_{n}
\end{aligned}
$$

We have the equality 


$$
L^{1}\left(X_{N}, \mu_{N}\right)=\otimes_{i=1}^{n} L^{1}\left(X_{i}, \mu_{i}\right) .
$$

In effect, the set of simple integrable functions is dense in $L^{1}\left(X_{N}, \mu_{N}\right)$ and any simple integrable function can be approximated in $L^{1}\left(X_{N}, \mu_{N}\right)$ by finite linear combinations of characteristic functions of the type $\chi_{E_{N}}=$ $\otimes_{i=1}^{n} \chi_{E_{i}}$ with $E_{i} \subseteq X_{i}, \mu_{i}\left(E_{i}\right)<\infty$ (this last fact can be deduced from the definition of product measure).

From Theorem 2.1 and (2.2) we obtain a decomposition of $L^{1}\left(X_{N}, \mu_{N}\right)$ given in the next theorem.

Theorem 2.2. 2.2. Let $g_{i} \in L^{1}\left(X_{i}, \mu_{i}\right)$ with $\int_{X_{i}} g_{i}=1, i \in N$. Then

$$
L^{1}\left(X_{N}, \mu_{N}\right)=\oplus_{K \subseteq N} \otimes_{k \in K}\left\langle g_{k}\right\rangle \otimes_{k^{\prime} \in N \backslash K} L_{0}^{1}\left(X_{k^{\prime}}, \mu_{k^{\prime}}\right) .
$$

For a detailed proof of the above theorem see Morillas (2003).

\section{Density functions in $L^{1}\left(X_{N}, \mu_{N}\right)$ with given unidimensional marginals.}

If $\emptyset \subset K \subseteq N$, then the set of density functions in $L^{1}\left(X_{K}, \mu_{K}\right)$ is

$$
\widetilde{X_{K}}=\left\{f \in L^{1}\left(X_{K}, \mu_{K}\right): f \geq 0\right.
$$

and

$$
\left.\int_{X_{K}} f d \mu_{K}=1\right\}
$$

For $i \in N$, consider the continuous linear function

$$
\begin{array}{r}
s_{i}: L^{1}\left(X_{N}, \mu_{N}\right) \rightarrow L^{1}\left(X_{i}, \mu_{i}\right) \\
f \rightarrow \int_{X_{N \backslash\{i\}}} f d \mu_{N \backslash\{i\}} .
\end{array}
$$

If $g_{i} \in \widetilde{X}_{i}, i \in N$, then the set of density functions in $L^{1}\left(X_{N}, \mu_{N}\right)$ with unidimensional marginals $g_{i}$ is

$$
U\left(g_{N}\right)=\left\{f \in \widetilde{X_{N}}: s_{i}(f)=g_{i}, i \in N\right\} .
$$

In this section we are going to give an analytical description of $U\left(g_{N}\right)$. In what follows we will suppose that for $i \in N, g_{i}, h_{i} \in L^{1}\left(X_{i}, \mu_{i}\right)$ and $\int_{X_{i}} g_{i} d \mu_{i}=\int_{X_{i}} h_{i} d \mu_{i}=1$. Next we will consider some functions introduced 
by Marchi (1972) for the case in which $X_{i}$ are finite sets. We begin with the continuous linear function

$$
\begin{array}{r}
s_{N}: L^{1}\left(X_{N}, \mu_{N}\right) \rightarrow \times_{i=1}^{n} L^{1}\left(X_{i}, \mu_{i}\right) \\
f \rightarrow\left(s_{1}(f), \ldots, s_{n}(f)\right)
\end{array}
$$

By Theorem 2.1, $L^{1}\left(X_{i}, \mu_{i}\right)=L_{0}^{1}\left(X_{i}, \mu_{i}\right) \oplus\left\langle h_{i}\right\rangle$. Thus, if we consider the functions associated with this topological sum, $\sigma_{i}$ given by (2.1) and the projection $p_{i}$ given by

$$
\begin{array}{r}
p_{i}: L_{0}^{1}\left(X_{i}, \mu_{i}\right) \times\left\langle h_{i}\right\rangle \rightarrow L_{0}^{1}\left(X_{i}, \mu_{i}\right) \\
\left(z, c h_{i}\right) \rightarrow z,
\end{array}
$$

then the function

$$
\begin{aligned}
u_{i}: L^{1}\left(X_{i}, \mu_{i}\right) & \rightarrow L_{0}^{1}\left(X_{i}, \mu_{i}\right) \\
f & \rightarrow p_{i} \sigma_{i}^{-1}(f),
\end{aligned}
$$

is a continuous linear transformation. If $f \in L^{1}\left(X_{N}, \mu_{N}\right)$, then

$$
\begin{aligned}
s_{i}(f)= & u_{i}\left(s_{i}(f)\right)+\left(\int_{X_{i}} s_{i}(f) d \mu_{i}\right) h_{i} \\
& =u_{i}\left(s_{i}(f)\right)+\left(\int_{X_{N}} f d \mu_{N}\right) h_{i}
\end{aligned}
$$

Consider now the function

$$
\begin{aligned}
p_{h_{i}}: L^{1}\left(X_{i}, \mu_{i}\right) & \rightarrow L^{1}\left(X_{i}, \mu_{i}\right) \\
f & \rightarrow u_{i}(f)+h_{i} .
\end{aligned}
$$

Let

$$
\begin{array}{r}
\left.M_{i}: L^{1}\left(X_{i}, \mu_{i}\right)=\operatorname{range}\left(p_{h_{i}}\right) \mu_{i}\right) \\
=\left\{f \in L^{1}\left(X_{i}, \mu_{i}\right): \int_{X_{i}} f d \mu_{i}=1\right\} \\
=\left\{z+h_{i}: z \in L_{0}^{1}\left(X_{i}, \mu_{i}\right)\right\},
\end{array}
$$

and

$$
\mathcal{M}=\left\{f \in L^{1}\left(X_{N}, \mu_{N}\right): \int_{X_{N}} f d \mu_{N}=1\right\} .
$$

From (3.1), if $f \in \mathcal{M}$ then

$$
p_{h_{i}} s_{i}(f)=s_{i}(f) .
$$

Let

$$
\begin{array}{r}
p_{h_{N}}: \times_{i=1}^{n} L^{1}\left(X_{i}, \mu_{i}\right) \rightarrow \times_{i=1}^{n} L^{1}\left(X_{i}, \mu_{i}\right) \\
\left(f_{N}\right) \rightarrow\left(p_{h_{1}}\left(f_{1}\right), \ldots, p_{h_{n}}\left(f_{n}\right)\right),
\end{array}
$$




$$
\begin{array}{r}
\left.\Psi: \times_{i=1}^{n} L^{1}\left(X_{i}, \mu_{i}\right)\left(f_{n}\right)\right), \underset{L^{1}\left(X_{N}, \mu_{N}\right)}{ } \\
\left(f_{N}\right) \rightarrow \otimes_{i=1}^{n} f_{i}
\end{array}
$$

and

$$
\begin{gathered}
\pi_{h_{N}}: L^{1}\left(X_{N}, \mu_{N}\right) \rightarrow L^{1}\left(X_{N}, \mu_{N}\right) \\
f \rightarrow \Psi p_{h_{N}} s_{N}(f)
\end{gathered}
$$

Next theorem asserts that $U\left(g_{N}\right)$ consists of the nonnegative elements of $\mathcal{M} \cap \pi_{h_{N}}^{-1}\left(\Psi\left(g_{N}\right)\right)$.

Theorem 3.1. 3.1. $\mathcal{M} \cap \pi_{h_{N}}^{-1}\left(\Psi\left(g_{N}\right)\right)=\left\{f \in L^{1}\left(X_{N}, \mu_{N}\right): s_{i}(f)=g_{i}, i \in\right.$ $N\}$.

Proof. Suppose that $f \in \mathcal{M}$. Using (3.4) we obtain

$$
\begin{aligned}
\pi_{h_{N}} & \left.=\Psi p_{h_{N}} s_{N}(f)\right) . \\
& =\Psi p_{h_{N}}\left(s_{1}(f), \ldots, s_{n}(f)\right) \\
& =\Psi\left(s_{1}(f), \ldots, s_{n}(f)\right) \\
& =\Psi\left(s_{N}(f)\right)
\end{aligned}
$$

By (3.6), if $f \in \mathcal{M} \cap \pi_{h_{N}}^{-1}\left(\Psi\left(g_{N}\right)\right)$, then

$$
\Psi\left(s_{N}(f)\right)=\Psi\left(g_{N}\right) .
$$

Taking into account that $\int_{X_{i}} s_{i}(f) d \mu_{i}=\int_{X_{N}} f d \mu_{N}=1$ and $\int_{X_{i}} g_{i} d \mu_{i}=$ 1 , for all $i \in N$, from the previous equality it results that if $i \in N$, then

$$
\begin{array}{r}
s_{i}(f)=s_{i}\left(\Psi\left(s_{N}(f)\right)\right) \\
=s_{i}\left(\Psi\left(g_{N}\right)\right) \\
=g_{i} .
\end{array}
$$

Suppose now that for each $i \in N, s_{i}(f)=g_{i}$. Then,

$$
\operatorname{int}_{X_{N}} f d \mu_{N}=\int_{X_{i}} s_{i}(f) d \mu_{i}=\int_{X_{i}} g_{i} d \mu_{i}=1
$$

and

$$
\Psi\left(s_{N}(f)\right)=\Psi\left(g_{N}\right) .
$$

Thus $f \in \mathcal{M}$ and, by $(3.6), \pi_{h_{N}}(f)=\Psi\left(g_{N}\right)$. 
Remark 3.2. Observe that if $f \in L^{1}\left(X_{N}, \mu_{N}\right)$, by (3.1)

$$
\begin{aligned}
\pi_{h_{N}}(f)= & \pi_{h_{N}}(f) s_{N}(f) \\
= & \Psi p_{h_{N}}\left(s_{1}(f), \ldots, s_{n}(f)\right) \\
= & \Psi p_{h_{N}}\left(u_{1}\left(s_{1}(f)\right)+\left(\int_{X_{N}} f d \mu_{N}\right) h_{1}, \ldots, u_{n}\left(s_{n}(f)\right)\right. \\
& \left.+\left(\int_{X_{N}} f d \mu_{N}\right) h_{n}\right) \\
= & \Psi\left(u_{1}\left(s_{1}(f)\right)+h_{1}, \ldots, u_{n}\left(s_{n}(f)\right)+h_{n}\right) \\
= & \sum_{K} \subseteq N
\end{aligned}
$$

By (3.1), if $s_{i}(f)=\left(\int_{X_{N}} f d \mu_{N}\right) h_{i}, i \in N$, then $u_{i}\left(s_{i}(f)\right)=0$ and, by (3.7), $\pi_{h_{N}}(f)=\Psi\left(h_{N}\right)$.

On the other hand, since $\int_{X_{k}} h_{k} d \mu_{k}=1$ and $u_{k}\left(s_{k}(f)\right) \in L_{0}^{1}\left(X_{k}, \mu_{k}\right)$, $k \in N$, from (3.7) we deduce that

$$
s_{i}\left(\pi_{h_{N}}(f)\right)=u_{i}\left(s_{i}(f)\right)+h_{i} .
$$

If $\pi_{h_{N}}(f)=\Psi\left(h_{N}\right)$, then for each $i \in N, s_{i}\left(\pi_{h_{N}}(f)\right)=h_{i}$; and from the above equality, $u_{i}\left(s_{i}(f)\right)=0$, so, by $(3.1), s_{i}(f)=\left(\int_{X_{N}} f d \mu_{N}\right) h_{i}$.

From the previous observations we have,

$$
(3.8\rangle_{h_{N}}^{-1}\left(\Psi\left(h_{N}\right)\right)=\left\{f \in L^{1}\left(X_{N}, \mu_{N}\right): s_{i}(f)=\left(\int_{X_{N}} f d \mu_{N}\right) h_{i}, i \in N\right\} .
$$

In the sequel we will need the following results which proofs can be found in Morillas (2003).

Lemma 3.2. 3.3. Let $g_{i} \in L^{1}\left(X_{i}, \mu_{i}\right)$ with $\int_{X_{i}} g_{i} d \mu_{i}=1, i \in N$. The following identities hold:

1. $\otimes_{i=1}^{n}\left\langle g_{i}\right\rangle=\left\langle\otimes_{i=1}^{n} g_{i}\right\rangle$.

2. $\otimes_{k \in N \backslash\{i\}}\left\langle g_{k}\right\rangle \otimes L_{0}^{1}\left(X_{i}, \mu_{i}\right)=\left\{\otimes_{k \in N \backslash\{i\}} g_{k} \otimes z_{i}: z_{i} \in L_{0}^{1}\left(X_{i}, \mu_{i}\right)\right\}$.

3. If $K \subseteq N,|K| \geq 2$, then

$$
\begin{aligned}
& \otimes_{k \in N \backslash K}\left\langle g_{k}\right\rangle \otimes_{k^{\prime} \in K} L_{0}^{1}\left(X_{k^{\prime}}, \mu_{k^{\prime}}\right)= \\
& =c l\left\{\otimes_{k \in N \backslash K} g_{k} \otimes \sum_{l=1}^{m} \otimes_{k^{\prime} \in K} z_{k^{\prime}}^{l}: z_{k^{\prime}}^{l} \in L_{0}^{1}\left(X_{k^{\prime}}, \mu_{k^{\prime}}\right), k^{\prime} \in K, l \in\right. \\
& \{1, \ldots, m\}, m \in \mathbf{N}\} .
\end{aligned}
$$


Lemma 3.3. 3.4. Let $g_{i} \in L^{1}\left(X_{i}, \mu_{i}\right)$ with $\int_{X_{i}} g_{i} d \mu_{i}=1, i \in N$. Let $\emptyset \subset K \subseteq N, \emptyset \subseteq J \subseteq N$ and $f_{N \backslash K} \in \otimes_{k \in N \backslash K}\left\langle g_{k}\right\rangle \otimes_{k^{\prime} \in K} L_{0}^{1}\left(X_{k^{\prime}}, \mu_{k^{\prime}}\right)$. The following assertions hold:

1. If $J=K$, then $\int_{X_{N \backslash J}} f_{N \backslash K} d \mu_{N \backslash J} \in \otimes_{k^{\prime} \in K} L_{0}^{1}\left(X_{k^{\prime}}, \mu_{k^{\prime}}\right)$.

2. If $J \neq K$ and $|K| \geq|J|$, then $\int_{X_{N \backslash J}} f_{N \backslash K} d \mu_{N \backslash J}=0$.

Now are in conditions to give an analytical description of the set $\mathcal{M} \cap$ $\pi_{h_{N}}^{-1}\left(\Psi\left(g_{N}\right)\right)$.

Theorem 3.4. 3.5. $\mathcal{M} \cap \pi_{h_{N}}^{-1}\left(\Psi\left(g_{N}\right)\right)=\left\{f \in L^{1}\left(X_{N}, \mu_{N}\right): f=\Psi\left(g_{N}\right)+\right.$ $\left.r, r \in \oplus_{K \subseteq N,|K| \geq 2} \otimes_{k \in N \backslash K}\left\langle g_{k}\right\rangle \otimes_{k^{\prime} \in K} L_{0}^{1}\left(X_{k^{\prime}}, \mu_{k^{\prime}}\right)\right\}$.

Proof. Suppose that $f=\Psi\left(g_{N}\right)+r$ where $r \in \oplus_{K \subseteq N,|K| \geq 2} \otimes_{k \in N \backslash K}$ $\left\langle g_{k}\right\rangle \otimes_{k^{\prime} \in K} L_{0}^{1}\left(X_{k^{\prime}}, \mu_{k^{\prime}}\right)$. If $i \in N$, using Lemma 3.4 (2) with $J=\{i\}$, it results that $s_{i}(f)=g_{i}$. So, by Theorem 3.1, $f \in \mathcal{M} \cap \pi_{h_{N}}^{-1}\left(\Psi\left(g_{N}\right)\right)$.

Suppose now that $f \in \mathcal{M} \cap \pi_{h_{N}}^{-1}\left(\Psi\left(g_{N}\right)\right)$. By Theorem 2.2,

$$
f=c \Psi\left(g_{N}\right)+\sum_{K \subseteq N,|K| \geq 1} f_{N \backslash K},
$$

with $f_{N \backslash K} \in \otimes_{k \in N \backslash K}\left\langle g_{k}\right\rangle \otimes_{k^{\prime} \in K} L_{0}^{1}\left(X_{k^{\prime}}, \mu_{k^{\prime}}\right)$, for $K \subseteq N$. Using Lemma 3.4 (2) with $J=\emptyset$, it results $\int_{X_{N}} f d \mu_{N}=c$. Since $f \in \mathcal{M}$, then $c=1$. By Lemma 3.3 (2), for each $i \in N$, there exists $z_{i} \in L_{0}^{1}\left(X_{i}, \mu_{i}\right)$ such that $f_{N \backslash\{i\}}=\otimes_{k \in N \backslash\{i\}} g_{k} \otimes z_{i}$. If $i \in N$, by Lemma 3.2. with $J=\{i\}$ and (3.9) with $c=1$, we have

$$
s_{i}(f)=g_{i}+z_{i} .
$$

By Theorem 3.1, since $f \in \mathcal{M} \cap \pi_{h_{N}}^{-1}\left(\Psi\left(g_{N}\right)\right)$, then $s_{i}(f)=g_{i}$, and consequently $z_{i}=0$. Thus, for $i \in N, f_{N \backslash\{i\}}=0$, resulting for $f$ the expression that we like. 
Remark 3.6. Using (3.8) and a similar argument to that used in the previous proof, we obtain that

$$
\begin{array}{r}
\pi_{h_{N}}^{-1}\left(\Psi\left(h_{N}\right)\right)=\left\{f \in L^{1}\left(X_{N}, \mu_{N}\right): f=\left(\int_{X_{N}} f d \mu_{N}\right) \Psi\left(h_{N}\right)+r,\right. \\
\left.r \in \oplus_{K \subseteq N,|K| \geq 2} \otimes_{k \in N \backslash K}\left\langle h_{k}\right\rangle \otimes_{k^{\prime} \in K} L_{0}^{1}\left(X_{k^{\prime}}, \mu_{k^{\prime}}\right)\right\} .
\end{array}
$$

By Theorem Theorem 3.5,

$$
\begin{aligned}
& \mathcal{M} \cap \pi_{h_{N}}^{-1}\left(\Psi\left(g_{N}\right)\right)-\Psi\left(g_{N}\right)= \\
& \bigoplus_{K \subseteq N,|K| \geq 2} \otimes_{k \in N \backslash K}\left\langle g_{k}\right\rangle \otimes_{k^{\prime} \in K} L_{0}^{1}\left(X_{k^{\prime}}, \mu_{k^{\prime}}\right) .
\end{aligned}
$$

Corollary 3.1. Let $g_{i}^{\prime} \in L^{1}\left(X_{i}, \mu_{i}\right)$ be such that $\int_{X_{i}} g_{i}^{\prime} d \mu_{i}=1, i \in N$. Then $\mathcal{M} \cap \pi_{h_{N}}^{-1}\left(\Psi\left(g_{N}\right)\right)-\Psi\left(g_{N}\right)=\mathcal{M} \cap \pi_{h_{N}}^{-1}\left(\Psi\left(g_{N}^{\prime}\right)\right)-\Psi\left(g_{N}^{\prime}\right)$.

Proof. If $r \in \mathcal{M} \cap \pi_{h_{N}}^{-1}\left(\Psi\left(g_{N}\right)\right)-\Psi\left(g_{N}\right)$, then there exists $f \in \mathcal{M} \cap$ $\pi_{h_{N}}^{-1}\left(\Psi\left(g_{N}\right)\right)$ such that $r=f-\Psi\left(g_{N}\right)$. By Theorem 3.1, $s_{i}(f)=g_{i}$, so $f^{\prime}=r+\Psi\left(g_{N}^{\prime}\right)$ satisfies $s_{i}\left(f^{\prime}\right)=g_{i}^{\prime}$. Consequently, using again Theorem 3.1, $f^{\prime} \in \mathcal{M} \cap \pi_{h_{N}}^{-1}\left(\Psi\left(g_{N}^{\prime}\right)\right)$, and thus $r \in \mathcal{M} \cap \pi_{h_{N}}^{-1}\left(\Psi\left(g_{N}^{\prime}\right)\right)-\Psi\left(g_{N}^{\prime}\right)$. This shows that $\mathcal{M} \cap \pi_{h_{N}}^{-1}\left(\Psi\left(g_{N}\right)\right)-\Psi\left(g_{N}\right) \subseteq \mathcal{M} \cap \pi_{h_{N}}^{-1}\left(\Psi\left(g_{N}^{\prime}\right)\right)-\Psi\left(g_{N}^{\prime}\right)$. Since $\left(g_{N}\right)$ and $\left(g_{N}^{\prime}\right)$ are arbitrary, this shows that $\mathcal{M} \cap \pi_{h_{N}}^{-1}\left(\Psi\left(g_{N}\right)\right)-\Psi\left(g_{N}\right)=$ $\mathcal{M} \cap \pi_{h_{N}}^{-1}\left(\Psi\left(g_{N}^{\prime}\right)\right)-\Psi\left(g_{N}^{\prime}\right)$.

By the previous corollary and (3.10),

$$
\begin{aligned}
\oplus_{K \subseteq N,|K| \geq 2} \otimes_{k \in N \backslash K}\left\langle g_{k}\right\rangle \otimes_{k^{\prime} \in K} L_{0}^{1}\left(X_{k^{\prime}}, \mu_{k^{\prime}}\right)= & \\
& =\oplus_{K \subseteq N,|K| \geq 2} \otimes_{k \in N \backslash K}\left\langle g_{k}^{\prime}\right\rangle \otimes_{k^{\prime} \in K} L_{0}^{1}\left(X_{k^{\prime}}, \mu_{k^{\prime}}\right) .
\end{aligned}
$$

In what follows we will denote with $\mathcal{R}$ the set given by

$$
\begin{aligned}
& \mathcal{R}=\mathcal{M} \cap \pi_{h_{N}}^{-1}\left(\Psi\left(g_{N}\right)\right)-\Psi\left(g_{N}\right) \\
& \quad=\oplus_{K \subseteq N,|K| \geq 2} \otimes_{k \in N \backslash K}\left\langle g_{k}\right\rangle \otimes_{k^{\prime} \in K} L_{0}^{1}\left(X_{k^{\prime}}, \mu_{k^{\prime}}\right)
\end{aligned}
$$

The previous results tell us that the Banach space $\mathcal{R}(\mathcal{R}$ is a closed subspace of $\left.L^{1}\left(X_{N}, \mu_{N}\right)\right)$ do not depend of the functions $g_{i}$ nor of the functions $h_{i}$.

By the results of this section, when each $g_{i} \in \widetilde{X}_{i}$, by Theorem 3.1, $U\left(g_{N}\right)$ consists of the nonnegative elements of $\mathcal{M} \cap \pi_{h_{N}}^{-1}\left(\Psi\left(g_{N}\right)\right)$ whose analytical description is given by Theorem 3.5. Concretely,

$$
\begin{aligned}
& U\left(g_{N}\right)=\left\{f \in \mathcal{M} \cap \pi_{h_{N}}^{-1}\left(\Psi\left(g_{N}\right)\right): f \geq 0\right\} \\
& =\left\{\Psi\left(g_{N}\right)+r: r \in \mathcal{R} \text { and } \geq-\Psi\left(g_{N}\right)\right\} .
\end{aligned}
$$




\section{The natural vector bundle associated with $\widetilde{X_{N}}$}

In this section we are going to use some notions about differentiable manifolds (see e.g. Larotonda (1980)). For simplicity, we are going to use the term manifold as a synonym of $C^{\infty}$-manifold.

In order to obtain the vector bundle we introduce some differentiable structures.

Lemma 4.1. 4.1. Let $q$ be the continuous linear transformation defined by

$$
\begin{array}{r}
q: L^{1}(X, \mu) \rightarrow \mathbf{R} \\
f \rightarrow \int_{X} f d \mu .
\end{array}
$$

Then $q$ is a submersion.

Proof. Clearly $q$ is $C^{\infty}$. Since for all $f \in L^{1}(X, \mu), D q(f)=q$ is onto, then $\operatorname{Ker}(D q(f))=L_{0}^{1}(X, \mu)$ and, by Theorem 2.1, $L_{0}^{1}(X, \mu)$ has a topological supplement, then $D q(f)$ is a topological epimorphism. Thus $q$ is a submersion.

Proposition 4.2. 4.2. The set $\mathcal{M}$ given in (3.3) is a closed submanifold of $L^{1}\left(X_{N}, \mu_{N}\right)$ pure of model $L_{0}^{1}\left(X_{N}, \mu_{N}\right)$.

Proof. If $q$ is given by (??) with $\left(X_{N}, \mu_{N}\right)$ instead of $(X, \mu)$, then $\mathcal{M}=q^{-1}(\{1\}) \neq \emptyset$ and the proposition follows from Lemma ??.

In a similar manner it can be proved that $M_{i}$ (see (3.2) is a closed submanifold of $L^{1}\left(X_{i}, \mu_{i}\right)$ pure of model $L_{0}^{1}\left(X_{i}, \mu_{i}\right)$. We also consider the product manifold $M_{N}=\times_{i=1}^{n} M_{i}=\operatorname{range}\left(p_{h_{N}}\right)$ pure of model $\times_{i=1}^{n} L_{0}^{1}\left(X_{i}, \mu_{i}\right)$.

Lemma 4.3. 4.3. Let $\Psi$ be the continuous symmetric multilinear function given by (3.5). Then $\Psi \mid M_{N}: M_{N} \rightarrow L^{1}\left(X_{N}, \mu_{N}\right)$ is a regular immersion. 
Proof. Let $\left(f_{N}\right),\left(f_{N}^{\prime}\right) \in M_{N}$ such that $\Psi\left(f_{N}\right)=\Psi\left(f_{N}^{\prime}\right)$. For each $i \in N$,

$$
\int_{X_{N \backslash\{i\}}} \Psi\left(f_{N}\right) d \mu_{N \backslash\{i\}}=\int_{X_{N \backslash\{i\}}} \Psi\left(f_{N}^{\prime}\right) d \mu_{N \backslash\{i\}},
$$

or which is the same, $f_{i}=f_{i}^{\prime}$. Then, $\left(f_{N}\right)=\left(f_{N}^{\prime}\right)$. This shows that $\Psi \mid M_{N}$ is one to one.

Since

$$
\begin{aligned}
& \left\|\Psi\left(f_{N}\right)-\Psi\left(f_{N}^{\prime}\right)\right\|_{\left(X_{N}, \mu_{N}\right)}= \\
& =\left\|\Psi\left(f_{N}\right)-f_{i} \otimes_{k \in N \backslash\{i\}} f_{k}^{\prime}+f_{i} \otimes_{k \in N \backslash\{i\}} f_{k}^{\prime}-\Psi\left(f_{N}^{\prime}\right)\right\|_{\left(X_{N}, \mu_{N}\right)} \\
& \leq\left\|f_{i}\right\|_{\left(X_{i}, \mu_{i}\right)}\left\|\otimes_{k \in N \backslash\{i\}} f_{k}-\otimes_{k \in N \backslash\{i\}} f_{k}^{\prime}\right\|_{\left(X_{N \backslash\{i\}}, \mu_{N \backslash\{i\}}\right)}+ \\
& +\left\|f_{i}-f_{i}^{\prime}\right\|_{\left(X_{i}, \mu_{i}\right)}\left\|\otimes_{k \in N \backslash\{i\}} f_{k}^{\prime}\right\|_{\left(X_{N \backslash\{i\}}, \mu_{N \backslash\{i\}}\right)},
\end{aligned}
$$

it results that $\Psi$ is continuous.

If $\left(f_{N}\right),\left(f_{N}^{\prime}\right) \in M_{N}$, we have

$$
\int_{X_{N \backslash\{i\}}} \otimes_{k \in N \backslash\{i\}} f_{k} d \mu_{N \backslash\{i\}}=\int_{X_{N \backslash\{i\}}} \otimes_{k \in N \backslash\{i\}} f_{k}^{\prime} d \mu_{N \backslash\{i\}}=1
$$

for each $i \in N$. Therefore

$$
\begin{aligned}
\left\|f_{i}-f_{i}^{\prime}\right\|_{\left(X_{i}, \mu_{i}\right)} & = \\
& =\int_{X_{i}} \mid f_{i} \int_{X_{N \backslash\{i\}}} \otimes_{k \in N \backslash\{i\}} f_{k} d \mu_{N \backslash\{i\}} \\
& =-f_{i}^{\prime} \int_{X_{N \backslash\{i\}}} \otimes_{k \in N \backslash\{i\}} f_{k}^{\prime} d \mu_{N \backslash\{i\}} \mid d \mu_{i} \\
& =\left\|\Psi\left(f_{N}\right)-\Psi\left(f_{N}^{\prime}\right)\right\|_{\left(X_{N}, \mu_{N}\right)} .
\end{aligned}
$$

Thus, $\Psi \mid M_{N}$ is a topological isomorphism from $M_{N}$ to $\Psi\left(M_{N}\right)$.

Since $\Psi$ is a continuous symmetric multilinear function then it is $C^{\infty}$ and its differential is given by

$$
D \Psi\left(g_{N}\right)\left(z_{N}\right)=\sum_{i=1}^{n} \Psi\left(z_{i}, g_{N \backslash\{i\}}\right)
$$


for all $\left(g_{N}\right),\left(z_{N}\right) \in \times_{i=1}^{n} L^{1}\left(X_{i}, \mu_{i}\right)$. Let $\left(g_{N}\right) \in M_{N},\left(z_{N}\right) \in \times_{i=1}^{n} L_{0}^{1}\left(X_{i}, \mu_{i}\right)$ be such that $D \Psi\left(g_{N}\right)\left(z_{N}\right)=0$. For each $k \in N$,

$$
\begin{array}{r}
z_{k}=\sum_{i=1}^{n} \int_{X_{N \backslash\{k\}}} \Psi\left(z_{i}, g_{N \backslash\{i\}}\right) d \mu_{N \backslash\{k\}} \\
=\int_{X_{N \backslash\{k\}}} D \psi\left(g_{N}\right)\left(z_{N}\right) d \mu_{N \backslash\{k\}} \\
=0 .
\end{array}
$$

Then $\left(z_{N}\right)=0$. Therefore, for each $\left(g_{N}\right) \in M_{N}, D \Psi\left(g_{N}\right) \mid \times_{i=1}^{n} L_{0}^{1}\left(X_{i}, \mu_{i}\right)$ is one to one.

We also have, from Lemma 3.3 and Theorem 2.2, that for each $\left(g_{N}\right) \in$ $M_{N}$, the set

$$
\begin{aligned}
& \operatorname{range}\left(D \Psi\left(g_{N}\right) \mid \times_{i=1}^{n} L_{0}^{1}\left(X_{i}, \mu_{i}\right)\right) \\
& =\left\{\sum_{i=1}^{n} \Psi\left(z_{i}, g_{N \backslash\{i\}}\right): z_{i} \in L_{0}^{1}\left(X_{i}\right) \text { for } i \in N\right\} \\
& =\oplus_{i=1}^{n} \otimes_{k \in N \backslash\{i\}}\left\langle g_{k}\right\rangle \otimes L_{0}^{1}\left(X_{i}, \mu_{i}\right),
\end{aligned}
$$

is a direct subspace of $L^{1}\left(X_{N}, \mu_{N}\right)$.

Consequently, for each $\left(g_{N}\right) \in M_{N}, D \Psi\left(g_{N}\right) \mid \times_{i=1}^{n} L_{0}^{1}\left(X_{i}, \mu_{i}\right)$ is a direct monomorphism.

Since $\Psi \mid M_{N}$ is a topological isomorphism from $M_{N}$ to $\Psi\left(M_{N}\right), \Psi$ is $C^{\infty}$ and for each $\left(g_{N}\right) \in M_{N}, D \Psi\left(g_{N}\right) \mid \times_{i=1}^{n} L_{0}^{1}\left(X_{i}, \mu_{i}\right)$ is a direct monomorphism, then $\Psi$ is a regular immersion.

As a consequence of the previous lemma we have

Proposition 4.4. 4.4. $\Psi\left(M_{N}\right)$ is a submanifold of $L^{1}\left(X_{N}, \mu_{N}\right)$ and $\Psi \mid M_{N}$ : $M_{N} \rightarrow \Psi\left(M_{N}\right)$ is a $C^{\infty}$-isomorphism.

In the sequel we will think about $\Psi \mid M_{N}$ as a function from $M_{N}$ onto $\Psi\left(M_{N}\right)$ and we will consider in $\mathcal{M} \cap \pi_{h_{N}}^{-1}\left(\Psi\left(g_{N}\right)\right)$ the structure of normed vectorial space that makes the map

$$
\begin{array}{r}
T: \mathcal{M} \cap \pi_{h_{N}}^{-1}\left(\Psi\left(g_{N}\right)\right) \rightarrow \mathcal{R} \\
f \rightarrow f-\Psi\left(g_{N}\right)
\end{array}
$$

a topological isomorphism. With this structure, $\mathcal{M} \cap \pi_{h_{N}}^{-1}\left(\Psi\left(g_{N}\right)\right)$ is a Banach space.

Now we are in condition to state and prove the main result of this section. 
Theorem 4.5. 4.5. $\left(\Psi\left(M_{N}\right), \mathcal{M}, \pi_{h_{N}} \mid \mathcal{M}\right)$ is a vector bundle with type fiber $\mathcal{R}$.

Proof. Since $s_{N}: L^{1}\left(X_{N}, \mu_{N}\right) \rightarrow \times_{i=1}^{n} L^{1}\left(X_{i}, \mu_{i}\right)$ is $C^{\infty}$, then $s_{N} \mid \mathcal{M}$ : $\mathcal{M} \rightarrow M_{N}$ is $C^{\infty}$. We also have that $\Psi: M_{N} \rightarrow \Psi\left(M_{N}\right)$ is $C^{\infty}$ and $\pi_{h_{N}}\left|\mathcal{M}=\Psi p_{h_{N}} s_{N}\right| \mathcal{M}=\Psi s_{N} \mid \mathcal{M}$. Then, $\pi_{h_{N}} \mid \mathcal{M}: \mathcal{M} \rightarrow \Psi\left(M_{N}\right)$ is $C^{\infty}$, because is the composition of $C^{\infty}$ functions.

Now define

$$
\begin{array}{r}
\varphi_{h_{N}}:\left(\pi_{h_{N}} \mid \mathcal{M}\right)^{-1}\left(\Psi\left(M_{N}\right)\right) \rightarrow \Psi\left(M_{N}\right) \times \mathcal{R} \\
\Psi\left(g_{N}\right)+r \rightarrow\left(\Psi\left(g_{N}\right), r\right) .
\end{array}
$$

Clearly, $\varphi_{h_{N}}$ is a $C^{\infty}$-isomorphism and

$$
\begin{array}{r}
p_{\Psi\left(M_{N}\right)} \varphi_{h_{N}}\left(\Psi\left(g_{N}\right)+r\right)=\Psi\left(g_{N}\right) \\
=\left(\pi_{h_{N}} \mid M\right)\left(\Psi\left(g_{N}\right)\right)
\end{array}
$$

where $p_{\Psi\left(M_{N}\right)}: \Psi\left(M_{N}\right) \times \mathcal{R} \rightarrow \Psi\left(M_{N}\right)$ is the projection. Taking into in account the structure of normed vectorial space defined in $\mathcal{M} \cap \pi_{h_{N}}^{-1}\left(\Psi\left(g_{N}\right)\right)=$ $\left(\pi_{h_{N}} \mid \mathcal{M}\right)^{-1}\left(\Psi\left(g_{N}\right)\right)$, this set is a Banach space and the map

$$
\begin{array}{r}
\left(\pi_{h_{N}} \mid \mathcal{M}\right)^{-1}\left(\Psi\left(g_{N}\right)\right) \rightarrow \\
f \rightarrow f-\Psi\left(g_{N}\right)
\end{array}
$$

is a topological isomorphism.

By the results of the previous section, if for each $i \in N, g_{i} \in \widetilde{X}_{i}$, then $U\left(g_{N}\right)$ consists of the nonnegative elements of the fiber $\left(\pi_{h_{N}} \mid \mathcal{M}\right)^{-1}\left(\Psi\left(g_{N}\right)\right)$. Also, any density function $f$ in $L^{1}\left(X_{N}, \mu_{N}\right)$ with unidimensional marginals $g_{i}$, can be written as $f=\Psi\left(g_{N}\right)+r$ where $r$ is in the type fiber $\mathcal{R}$.

\section{Concluding remarks.}

If the sets $X_{i}$ are finite and we consider on them the cardinal measure, the above results reduce to those in Marchi (1972). As we have mentioned in the introduction, in the discrete case they motivate a great variety of new concepts of cooperative equilibrium in game theory. Now we could use the results obtained in this paper to extend all these concepts for continuous games. On the other hand, there are several properties of the derived fiber 
bundle that can be studied. For example, problems concerning perturbation of the fibers and cross sections.

It is important to note that the set of density functions with given marginals appears in the study of the optimal transportation problem (see, e. g., Cafarelli, Feldman and McCann (2001), Cafarelli (2003)). More recently, it also appears in conexion with the application of game theory to physics, in particular, to quantum mechanics (see, e.g., Landsburg and references therein). We think that the results obtained here provide new geometrical tools to study all these matters.

Acknowledgement : We are grateful to Prof. Lizzetta Bruschi for some received help in the initial stage of this research.

\section{References}

[1] Cafarelli L., Feldman M. and McCann R. J. - Constructing optimal maps for Mongeś transport problem as a limit of strictly convex costs, Journal of the American Mathematical Society, Vol. 15, No. 1, pp. $1-26,(2001)$.

[2] Cafarelli L. - The Monge-Ampére equation and optimal transportation, an elementary review, Lectures Notes in Mathematics, Vol. 1813, Springer-Verlag, pp. 1-10, (2003).

[3] DallÁglio G. - Fréchet classes: the beginnings, Advances in Probability Distributions with Given Marginals, Eds. G. DallÁglio, S. Kotz, and G. Salinetti. Kluwer Academic Publishers, Dordrecht, pp. 1-12, (1991).

[4] Fréchet, M. - Sur les tableaux de corrélation dont les marges sont données. Ann. Univ. Lyon, Sc., Vol. 4, pp. 53-84, (1951).

[5] Hoeffding, W. Masstabinvariante korrelationstheorie, Schriften des Matematischen Instituts und des Instituts für Angewandte Mathematik der Universität Berlin, Vol. 5, Heft 3, pp. 179-233, (1940).

[6] Hoeffding, W. - Masstabinvariante korrelationsmasse für diskontinuierliche Verteilungen, Arkiv für mathematischen Wirtschaften und Sozialforschung, Vol. 7, pp. 43-70, (1941). 
[7] Joe, H. - Multivariate Models and Dependence Concepts, Chapman \& Hall, London, (1997).

[8] Larotonda A. - Notas de Variedades Diferenciables, INMABB, Universidad Nacional de Bahía Blanca, Argentina, (1980).

[9] Landsburg S. - Nash equilibria in quantum games, preprint available at http://www.landsburg.com/pdf.

[10] Marchi, E., García Jurado I. and Prada J. M. - Refinamientos del concepto de equilibrio en extensiones generalizadas de juegos finitos, Investigación Operativa, Vol. 6, No. 1, pp. 83-92, (1991).

[11] Marchi, E. - Cooperative equilibrium, Compt. \& Math. with Appl., Vol. 12B, No. 5/6, pp. 1185-1186, (1986a).

[12] Marchi, E. - Equilibrium points for rational games related with partitioned expanding economies, Advances in Modeling \& Simulation, Vol. 32, No. 2, pp. 57-64, (1992).

[13] Marchi, E. - Nucleolus, equilibria, correlation, Advances in Modeling \& Simulation Modeling, Vol. 32, No. 2, pp. 7-11, (1996).

[14] Marchi, E. - On the possibility of an unusual extension of the minimax theorem, Z. Wahrsch. Verw. Geb., Vol. 12, pp. 224-270, (1969).

[15] Marchi, E. - Some topics on equilibria, Transactions of the American Mathematical Society, Vol. 220, No. 493, pp. 87-102, (1976).

[16] Marchi, E. - The natural vector bundle of the set of product probability, Z. Wahrsch. Verw. Geb., Vol. 23, pp. 7-17, (1972).

[17] Marchi, E. - Toward some steps in game theory, Japan Journal of Applied Math., Vol. 3, No. 2, pp. 343-355, (1986b).

[18] Marchi, E.: Una nota acerca de los puntos e-estables perfectos y propios, Collectanea Mathematica, Vol. 39, pp. 9-19, (1988).

[19] Morillas, P. M.: Nuevos tópicos en cópulas, funciones de densidad y un fibrado vectorial relacionado, Tesis doctoral, Universidad Nacional de San Luis, San Luis, Argentina, (2003).

[20] Nelsen, R. B.: An Introduction to Copulas, Lectures Notes in Statistics, Vol. 139, Springer-Verlag New York, Inc., (1999). 
[21] Schweizer, B. - Thirty years of copulas, Advances in Probability Distributions with Given Marginals, Eds. G. DallÁglio, S. Kotz, and G. Salinetti, Kluwer Academic Publishers, Dordrecht, pp. 13-50, (1991).

[22] Sklar, A. - Fonctions de répartition à n dimensions et leurs marges. Publ. Inst. Statist. Univ. Paris, Vol. 8, pp. 229-231, (1959).

\section{E. Marchi}

Instituto de Matemática Aplicada San Luis

UNSL-CONICET

Ejército de los Andes 950,

5700 San Luis

Argentina

e-mail : emarchi@unsl.edu.ar

and

P. M. Morillas

Instituto de Matemática Aplicada San Luis

UNSL-CONICET

Ejército de los Andes 950

5700 San Luis

Argentina

e-mail : morillas@unsl.edu.ar 